\title{
Challenges in diagnosis and treatment of melioidosis
}

\author{
TJJ Inglis 1 \\ Sri Lanka Journal of Infectious Diseases 2012 Vol.1(2); 52-53 \\ DOI: http://dx.doi.org/10.4038/sljid.v2i1.4067
}

Key words: pulmonary melioidosis; diagnosis; Burkholderia pseudomallei

This case report regarding a patient with pulmonary melioidosis illustrates the challenge posed when diagnosing and treating this infection. Even in melioidosis endemic areas the infection is less common than alternative diagnoses. ${ }^{1}$ Pulmonary lesions may mimic tuberculosis or even lung cancer, and thus lead the attending physician in the wrong direction. A further diagnostic difficulty is that confirmation of the infection is technically difficult. Serological studies provide no more than supportive evidence of prior exposure and false positives have been known to occur. Culture is ideal, but requires suitable selective agar for non-sterile sample types such as sputa or bronchoalveolar lavage fluid. Moreover, clinical laboratory staff must use a catalogue of procedures to identify the bacterial cause of melioidosis with confidence. ${ }^{2}$ The challenge does not diminish once the laboratory has confirmed melioidosis. Its bacterial cause, Burkholderia pseudomallei is usually resistant to Gentamicin and third generation cephalosporins such as Cefotaxime and Ceftriaxone. Extended spectrum cephalosporins, notably Ceftazidime, are effective but should be used for a prolonged period in conjunction with eradication therapy. ${ }^{3}$ The failure to respond to Meropenem therapy is an interesting observation that deserves further investigation. Some patients will succumb to melioidosis, despite optimal therapy. Other important aspects of clinical management include aggressive correction of hyperglycaemia, or any other metabolic anomaly caused by prior co-morbidities. Melioidosis is a good example of a complex infection that responds best to close collaboration between the physician and the pathologist. There is still a lot to be learned about its ideal clinical management.

${ }^{1}$ School of Pathology and Laboratory Medicine, University of Western Australia:

Address for correspondence: TJJ Inglis, DM, PhD, FRCPA, Professor (Pathology), School of Pathology and Laboratory Medicine, University of Western Australia.Email: tim.inglis@ health.wa.gov.au 


\section{References}

1. Cheng AC. Melioidosis: advances in diagnosis and treatment. Curr Opin Infect Dis. 2010; 23(6):554-9. doi:10.1097/QCO.0b013e32833fb88c

2. Inglis TJ, Rolim DB, Rodriguez JL. Clinical guideline for diagnosis and management of melioidosis. Rev Inst Med Trop São Paulo. 2006;48(1):1-4. doi:10.1590/S003646652006000100001

3. Inglis TJJ. The treatment of melioidosis. Pharmaceuticals 2010;3:1296-1303. doi:10.3390/ph3051296 